\title{
A coupled dual reciprocity BEM/Genetic algorithm for identification of blood perfusion parameters
}

\author{
Paul W. Partridge ${ }^{1 / 2}$ and Luiz C. Wrobel ${ }^{1}$ \\ ${ }^{1}$ School of Engineering and Design, Brunel University, \\ Uxbridge, Middlesex UB8 3PH, UK \\ ${ }^{2}$ On leave from Dept. Eng. Civil e Ambiental, Universidade de Brasilia, \\ 70910-900 Brasilia DF, Brazil
}

\begin{abstract}
Purpose - The paper presents an inverse analysis procedure based on a coupled numerical formulation through which the coefficients describing non-linear thermal properties of blood perfusion may be identified.

Design/methodology/approach - The coupled numerical technique involves a combination of the Dual Reciprocity Boundary Element Method and a Genetic Algorithm for the solution of the Pennes bioheat equation. Both linear and quadratic temperature-dependent variations are considered for the blood perfusion.

Findings - The proposed DRBEM formulation requires no internal discretisation and, in this case, no internal nodes either, apart from those defining the interface tissue/tumour. It is seen that the skin temperature variation changes as the blood perfusion increases, and in certain cases flat or nearly flat curves are produced. The proposed algorithm has difficulty to identify the perfusion parameters in these cases, although a more advanced GA may provide improved results.

Practical implications - The coupled technique allows accurate inverse solutions of the Pennes bioheat equation for quantitative diagnostics on the physiological conditions of biological bodies and for optimisation of hyperthermia for cancer therapy.

Originality/value - The proposed technique can be used to guide hyperthermia cancer treatment, which normally involves heating tissue to $42-43^{\circ} \mathrm{C}$. When heated up to this range of temperatures, the blood flow in normal tissues, e.g. skin and muscle, increases significantly, while blood flow in the tumour zone decreases. Therefore, the consideration of temperature-dependent blood perfusion in this case is not only essential for the correct modelling of the problem, but should provide larger skin temperature variations, making the identification problem easier
\end{abstract}

Keywords Boundary element method, Dual reciprocity method, Genetic algorithm, Inverse analysis, Tumours, Blood perfusion

Paper type Research paper 


\section{Introduction}

It is well known that the body surface temperature is controlled by blood circulation, local metabolism and heat transfer between the skin and the environment (Deng and Liu, 2004a). It is also known that several types of tumours, e.g. skin or breast, can lead to an increase in local blood flow, and thus to an increase in the local temperature (Liu and $\mathrm{Xu}, 2000$ ). On the other hand, thrombosis or vascular sclerosis decreases the blood flowing to the skin, resulting in lower skin temperatures (Liu and $\mathrm{Xu}, 2000$ ).

The Pennes bioheat equation can be used for the quantitative diagnostics of physiological conditions on biological bodies, e.g. for simulations of regional hyperthermia for cancer therapy (Tompkins et al., 1994; Erdmann et al., 1998; Lang et al., 1999). The parameters considered in Pennes' equation are usually assumed to be constant except for the blood perfusion, which varies with temperature (Tompkins et al., 1994; Erdmann et al., 1998; Lang et al., 1999; Rai and Rai, 1999; Liu and Xu, 2000). Herein, a numerical technique for identification of the temperature-dependent blood perfusion parameters in Pennes' equation is proposed based on the Dual Reciprocity Method (DRM), which has already been used for the direct solution of the bioheat equation (Deng and Liu, 2000; Deng and Liu, 2004a; Deng and Liu, 2004b; Lu et al., 1998; Liu and $\mathrm{Xu}, 2000)$. It is assumed that the size and location of the tumour are known from previous diagnostics; in the DRM, the tumour is treated as a sub-region and in addition to the nodes used to model the boundary of the tumour, no other internal points are required.

Previous works on inverse analysis of biological bodies were carried out by Ren et al. (1995), who applied the Boundary Element Method (BEM) to identify heat sources in biological bodies based on the simultaneous measurement of temperature and heat flux at the skin surface, by Majchrzak and Paruch (2004), who estimated the (constant) thermophysical parameters of a tumour using a least-squares algorithm based on sensitivity coefficients, and by Partridge and Wrobel (2007), who presented a BEM inverse analysis based on a Genetic Algorithm (GA) (Goldberg, 1989; Goldberg et al., 1997) to identify the position and size of shallow tumours using skin temperature measurements.

This paper extends the algorithm developed by Partridge and Wrobel (2007) for the identification of the coefficients of linear and quadratic variations of blood perfusion. A simple GA, as described in the literature (Castro and Partridge, 2006), is adequate for the problem. A cubic radial basis function is employed as an approximation function for the DRM, with linear augmentation (Golberg and Chen, 1994; Bridges and Wrobel, 1996; Partridge, 2000). Results of the evolution of the calculations using the GA are given for one of the cases considered.

The DRM formulation for the bioheat equation is considered in the next section. This is followed by some results of direct analyses, which illustrate the use of the DRM and the relationship between the parameters adopted to model blood perfusion and the temperature distribution on the skin surface. Then, the parameter identification problem is described and the results of some inverse analyses are presented, considering linear and quadratic expansions for the blood perfusion rate. The advantages and limitations of the proposed technique are also discussed.

\section{Application of the Dual Reciprocity Method to the Bioheat Equation}

The Pennes bioheat equation can be written in the following form (Deng and Liu, 2000; Deng and Liu, 2004a; Deng and Liu, 2004b; Lu et al., 1998; Liu and Xu, 2000), 


$$
\frac{\rho c}{k} \frac{\partial T}{\partial t}=\nabla^{2} T+\frac{\omega_{b} \rho_{b} c_{b}}{k}\left(T_{a}-T\right)+\frac{Q}{k}
$$

where $\rho, c$ and $k$ denote density, specific heat and thermal conductivity of tissue; $\rho_{b}, c_{b}$ are density and specific heat of blood, $\omega_{b}$ is the blood perfusion rate, $T_{a}$ is the arterial blood temperature and $Q$ is metabolic heating. Equation (1) is subject to the usual boundary conditions for thermal problems, (i) prescribed temperature $T=\bar{T}$; (ii) prescribed heat flux, $q=\bar{q}$. Equation (1) can be written, in steady-state form, as

$$
\nabla^{2} T=-\frac{\omega_{b} \rho_{b} c_{b}}{k}\left(T_{a}-T\right)-\frac{Q}{k}=b
$$

Equation (2) is a Poisson-type equation with two inhomogeneous terms, the first of which is dependent on the problem variable $T$, the other term being a function of space but not of the problem variable. Herein, this equation is solved using the DRM (Partridge et al., 1992) in which the fundamental solution to the Laplace equation, $u^{*}=1 / 2 \pi \log (1 / r)$, is employed to treat the term on the left-hand side of equation (2) and the inhomogeneous terms are taken to the boundary using the standard DRM (Partridge et al., 1992), leading to the system of equations:

$$
H T-G q=(H \hat{U}-G \hat{Q}) \alpha
$$

where the symbols have their usual meaning (Partridge et al., 1992). As the term $b$ in equation (2) is a function of the problem unknowns, equation (3) can be rewritten as

$$
H T-G q=(H \hat{U}-G \hat{Q}) F^{-1} b
$$

where the $F$ matrix is calculated from the definition of the approximating functions. Replacing $S=(H \hat{U}-G \hat{Q}) F^{-1}$ in equation (4), one obtains

$$
H T-G q=S b
$$

In the linear case (used here for the healthy tissue), it is possible to define $c_{1}=\omega_{b} \rho_{b} c_{b} / k$ and $c_{2}=-\left(\omega_{b} \rho_{b} c_{b} T_{a}+Q\right) / k$, and equation (5) can be written in the form:

$$
H T-G q=c_{1} S T+c_{2} S
$$

or

$$
\left(H-c_{1} S\right) T-G q=c_{2} S
$$

For a direct, well-posed problem, boundary conditions (temperature or heat flux) are applied to equation (7) in the usual way to produce 


$$
A x=y+d
$$

Equation (8) may finally be solved for the unknown boundary values contained in vector $x$.

Considering the temperature-dependence of the tumour's perfusion, $\omega_{b}$, the parameters $c_{1}$ and $c_{2}$ are redefined as follows:

$$
\begin{gathered}
c_{1}=c_{3} \omega_{b} \\
c_{2}=-\left(c_{4} \omega_{b}+\frac{Q}{k}\right)
\end{gathered}
$$

where $c_{3}=\rho_{b} c_{b} / k$ and $c_{4}=\rho_{b} c_{b} T_{a} / k$. When considering a quadratic variation for $\omega_{b}$,

$$
\omega_{b}=\left(\alpha+\beta T+\gamma T^{2}\right)
$$

parameters $c_{1}$ and $c_{2}$ assume the form

$$
\begin{gathered}
c_{1}=c_{3}\left(\alpha+\beta T+\gamma T^{2}\right) \\
c_{2}=-c_{4}\left(\alpha+\beta T+\gamma T^{2}\right)-\frac{Q}{k}
\end{gathered}
$$

Defining a diagonal matrix $R$ with the non-zero terms given by $R_{i}=\left(\alpha+\beta \bar{T}_{i}+\gamma \bar{T}_{i}^{2}\right)$, where $\bar{T}_{i}$ are known values of $T_{i}$ taken from a previous iteration and $i$ is the column and row number of the main diagonal, equation (11) can be rewritten as

$$
\begin{gathered}
c_{1}=c_{3} R \\
c_{2}=-\left(c_{4} R+\frac{Q}{k}\right)
\end{gathered}
$$

Replacing the above expressions into equation (6) finally gives

$$
H T-G q=c_{3} R S T-\left(c_{4} R+\frac{Q}{k}\right) S
$$

Taking the term with the unknown value of $T$ to the left-hand side in the usual way produces the final equation:

$$
\left(H-c_{3} R S\right) T-G q=-\left(c_{4} R+\frac{Q}{k}\right) S
$$

The above equation is solved by iterating on the values of $\bar{T}_{i}$ in matrix $R$. If a firstorder expansion is considered for $\omega_{b}, \gamma$ is equal to zero in equations (10) and (11). 
A cubic radial basis function, $r^{3}$, is used in the DRM approximation, with linear augmentation terms $1, x$ and $y$. The relevant particular solutions are given by (Golberg and Chen, 1994; Bridges and Wrobel, 1996; Partridge, 2000):

$$
f=r^{3}, \hat{u}=r^{5} / 25, f=1, \hat{u}=\left(x^{2}+y^{2}\right) / 4 ; f=x, \hat{u}=x^{3} / 6 ; f=y, \hat{u}=y^{3} / 6
$$

The above choice is justified by the results of the tests carried out by Partridge and Wrobel (2007) with several radial basis functions, including the 'classical' function $r$ and the thin plate spline $r^{2} \log r$, with and without augmentation. Results obtained using each of these functions were found to differ little. The tests in Partridge and Wrobel (2007) also showed that no internal points appear to be necessary in the DRM formulation for this type of problem. More details about the implementation of augmentation functions are given by Bridges and Wrobel (1996).

\section{Direct results for different values of the blood perfusion parameters}

Considering Figure 1, the external boundary, $\mathrm{ABCD}$ or $\Gamma_{2}$, is a vertical section through the skin tissue, the part $\mathrm{AD}$ being at the skin surface while the opposite boundary $\mathrm{BC}$ is considered to be an internal boundary maintained at body temperature, $T=37^{\circ} \mathrm{C}$. The boundaries $\mathrm{AB}$ and $\mathrm{CD}$ are truncation boundaries, at which the boundary condition $q=0 \mathrm{~W} / \mathrm{m}^{2}$ is considered. If the boundary $\mathrm{AD}$ is assumed to have a zero flux boundary condition, this is equivalent to thermal isolation on that boundary, for instance a bandage. The internal boundary or $\Gamma_{1}$ in Figure 1 is considered to divide the domain into two parts, $\Omega_{2}$ the external part and $\Omega_{1}$ which is a sub-region. On the boundary $\Gamma_{1}$ the usual compatibility and equilibrium conditions apply, i.e. $T_{1}=T_{2}$ and $q_{1}=-q_{2}$. The thickness of the skin is assumed to be $0.03 \mathrm{~m}$ and a section of length $0.08 \mathrm{~m}$ is considered. In what follows, the parameters $c_{1}$ and $c_{2}$ given in equation (6) are considered to be constant on $\Omega_{2}$, and taken to be the values for healthy tissue. However, in the sub-region $\Omega_{1}$, a nonlinear model is considered and different parameters are used for the blood perfusion, considering this sub-region to be a tumour. 


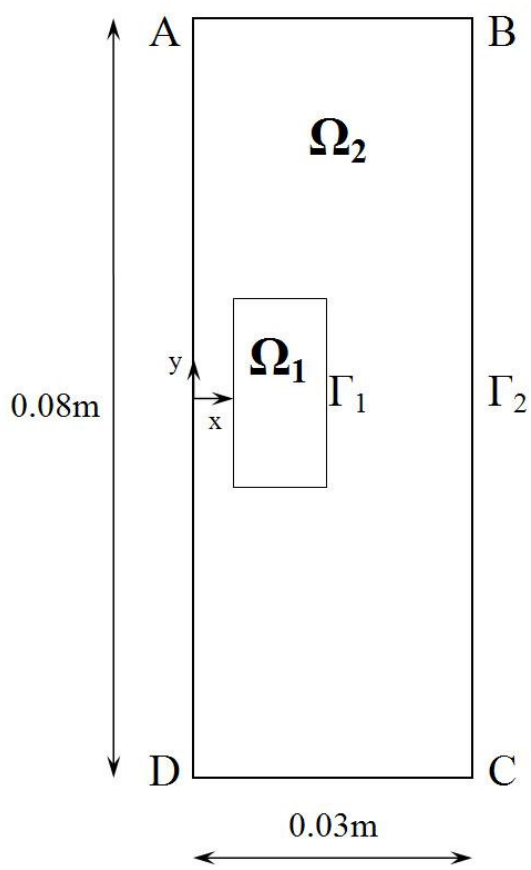

Figure 1. Tumour within a matrix of healthy tissue

\subsection{Results employing a first-order expansion}

With the boundary condition at the skin surface AD taken to be $q=0 \mathrm{~W} / \mathrm{m}^{2}$, values of the parameters necessary to calculate $c_{1}$ and $c_{2}$ for the healthy tissue are taken from Liu and $\mathrm{Xu}(2000): \rho_{b}=1000 \mathrm{~kg} / \mathrm{m}^{3}, c_{b}=4000 \mathrm{~J} /\left(\mathrm{kg}{ }^{\circ} \mathrm{C}\right), k=0.5 \mathrm{~W} /\left(\mathrm{m}{ }^{\circ} \mathrm{C}\right), \omega_{b}=$ $0.0005 \mathrm{ml}_{\mathrm{b}} / \mathrm{ml}_{\mathrm{t}} / \mathrm{s}$ and $Q=420 \mathrm{~J} /\left(\mathrm{m}^{3} \mathrm{~s}\right)$. For the tumour, the same values of $\rho_{b}, c_{b}$ and $k$ are taken, with $Q=4200 \mathrm{~J} /\left(\mathrm{m}^{3} \mathrm{~s}\right)$. The unit employed for the perfusion coefficient $\omega_{b}$ is such that $\rho_{b} \omega_{b}$ represents mass flow rate of blood per unit volume of tissue.

Initially, a first-order expansion for the blood perfusion is considered, $\omega_{b}=(\alpha+\beta T)$. Following Deng and Liu (2000), possible variations which the coefficients $\alpha$ and $\beta$ might take are as follows: (i) $\omega_{b}=0.0005+0.0001 T$, (ii) $\omega_{b}=0.005+0.0001 T$ and (iii) $\omega_{b}=0.005+0.0003 T$. According to equation (9), the parameter $\omega_{b}$ always appears multiplied by $\rho_{b} c_{b} / k$. Using the above numerical values gives the following expressions: (i) $c_{1}=800 \times(5+1 T)$, $c_{1}=800 \times(50+1 T)$ and (iii) $c_{1}=800 \times(50+3 T)$. Similar considerations are valid for the parameter $c_{2}$.

The discretisation adopted involves 16 linear elements along the internal boundary $\Gamma_{1}$ and 56 linear elements along $\Gamma_{2}$. Sensitivity tests were performed with different discretisations; for instance, using half the above number, i.e. 8 linear elements along $\Gamma_{1}$ and 28 linear elements along $\Gamma_{2}$, produces results which are virtually the same as those in Table 1 .

Results for different first-order variations for the tumour perfusion are given in Table 1. The table shows temperature values at the skin surface, boundary AD in Figure 1. It can be seen that the temperature variation in all cases is very small. The 
results for curves $\mathrm{B}, \mathrm{C}$ and $\mathrm{D}$ are flat or nearly so and the curvature changes for curves $\mathrm{E}$ and $\mathrm{F}$, in the sense that the temperatures at the centre points are lower than at the end points.

Since the temperature is almost constant in all cases, around $37^{\circ} \mathrm{C}$, the average value of the tumour perfusion for the linear variations considered are $\omega_{b}=0.00195$ $\mathrm{ml}_{\mathrm{b}} / \mathrm{ml}_{\mathrm{t}} / \mathrm{s}$ for curve A, $\omega_{b}=0.0042 \mathrm{ml}_{\mathrm{b}} / \mathrm{ml}_{\mathrm{t}} / \mathrm{s}$ for curve B, $\omega_{b}=0.00655 \mathrm{ml}_{\mathrm{b}} / \mathrm{ml}_{\mathrm{t}} / \mathrm{s}$ for curve $\mathrm{C}, \omega_{b}=0.094 \mathrm{ml}_{\mathrm{b}} / \mathrm{ml}_{\mathrm{t}} / \mathrm{s}$ for curve $\mathrm{D}, \omega_{b}=0.0141 \mathrm{ml}_{\mathrm{b}} / \mathrm{ml}_{\mathrm{t}} / \mathrm{s}$ for curve $\mathrm{E}$ and $\omega_{b}=$ $0.0161 \mathrm{ml}_{\mathrm{b}} / \mathrm{ml}_{\mathrm{t}} / \mathrm{s}$ for curve $\mathrm{F}$. The values for curves $\mathrm{D}, \mathrm{E}$ and $\mathrm{F}$ are high compared to average tumour perfusion values of $\omega_{b}=0.002 \mathrm{ml}_{\mathrm{b}} / \mathrm{ml}_{\mathrm{t}} / \mathrm{s}$ quoted in the literature (Liu and $\mathrm{Xu}, 2000$ ), explaining the unexpected results obtained with these curves.

Table 1: Temperature variations $\left({ }^{\circ} \mathrm{C}\right)$ at the skin surface considering first-order tumour perfusion

\begin{tabular}{|l|l|l|l|l|l|l|}
\hline $\begin{array}{l}y \text { at skin } \\
\text { surface }\end{array}$ & $\begin{array}{l}c_{1}=800 \times \\
(1+0.5 T) \\
\text { Curve A }\end{array}$ & $\begin{array}{l}c_{1}=800 \times \\
(5+1.0 T) \\
\text { Curve B }\end{array}$ & $\begin{array}{l}c_{1}=800 \times \\
(10+1.5 T) \\
\text { Curve C }\end{array}$ & $\begin{array}{l}c_{1}=800 \times \\
(20+2.0 T) \\
\text { Curve D }\end{array}$ & $\begin{array}{l}c_{1}=800 \times \\
(30+3.0 T) \\
\text { Curve E })\end{array}$ & $\begin{array}{l}c_{1}=800 \times \\
(50+3.0 T) \\
\text { Curve F }\end{array}$ \\
\hline 0.035 & 37.17 & 37.15 & 37.15 & 37.14 & 37.14 & 37.13 \\
\hline 0.03 & 37.18 & 37.15 & 37.15 & 37.14 & 37.13 & 37.13 \\
\hline 0.025 & 37.19 & 37.15 & 37.15 & 37.14 & 37.13 & 37.13 \\
\hline 0.02 & 37.21 & 37.15 & 37.15 & 37.14 & 37.12 & 37.12 \\
\hline 0.015 & 37.23 & 37.16 & 37.15 & 37.13 & 37.11 & 37.11 \\
\hline 0.01 & 37.27 & 37.16 & 37.15 & 37.13 & 37.10 & 37.09 \\
\hline 0.005 & 37.29 & 37.17 & 37.15 & 37.12 & 37.09 & 37.08 \\
\hline 0.0 & 37.30 & 37.17 & 37.15 & 37.12 & 37.09 & 37.08 \\
\hline-0.005 & 37.29 & 37.17 & 37.15 & 37.12 & 37.09 & 37.08 \\
\hline-0.01 & 37.27 & 37.16 & 37.15 & 37.13 & 37.10 & 37.09 \\
\hline-0.015 & 37.23 & 37.16 & 37.15 & 37.13 & 37.11 & 37.11 \\
\hline-0.02 & 37.21 & 37.15 & 37.15 & 37.14 & 37.12 & 37.12 \\
\hline-0.025 & 37.19 & 37.15 & 37.15 & 37.14 & 37.13 & 37.13 \\
\hline-0.03 & 37.18 & 37.15 & 37.15 & 37.14 & 37.13 & 37.13 \\
\hline-0.035 & 37.17 & 37.15 & 37.15 & 37.14 & 37.14 & 37.13 \\
\hline
\end{tabular}

\subsection{Results employing a second-order expansion}

Next, a second-order temperature-dependence for the tumour perfusion is considered. Results in Table 2 also show slight temperature variations at the skin surface, boundary $\mathrm{AD}$ in Figure 1, for all expansions adopted, which were chosen in order to cover a range of values similar to those in the first-order case. It can be seen that the results for curve $\mathrm{H}$ are flat and that for curves $\mathrm{I}$ and $\mathrm{J}$ have a different curvature from curve $\mathrm{G}$. The interpretation of these results is as discussed above. 
Table 2: Temperature variations $\left({ }^{\circ} \mathrm{C}\right)$ at the skin surface considering second-order tumour perfusion

\begin{tabular}{|c|c|c|c|c|}
\hline $\begin{array}{l}y \text { at } \\
\text { skin } \\
\text { surface }\end{array}$ & $\begin{array}{l}c_{1}=800 \times(3+ \\
\left.0.5 T+0.01 T^{2}\right) \\
\text { Curve G }\end{array}$ & $\begin{array}{l}c_{1}=800 \times(6+ \\
\left.1.0 T+0.002 T^{2}\right) \\
\text { Curve H }\end{array}$ & $\begin{array}{l}c_{1}=800 \times(2+ \\
\left.1.5 T+0.04 T^{2}\right) \\
\text { Curve I }\end{array}$ & $\begin{array}{l}c_{1}=800 \times(7+ \\
\left.1.9 T+0.07 T^{2}\right) \\
\text { Curve } \mathrm{J}\end{array}$ \\
\hline 0.035 & 37.16 & 37.15 & 37.14 & 37.14 \\
\hline 0.03 & 37.16 & 37.15 & 37.14 & 37.13 \\
\hline 0.025 & 37.17 & 37.15 & 37.13 & 37.13 \\
\hline 0.02 & 37.18 & 37.15 & 37.13 & 37.12 \\
\hline 0.015 & 37.19 & 37.15 & 37.12 & 37.11 \\
\hline 0.01 & 37.21 & 37.15 & 37.11 & 37.09 \\
\hline 0.005 & 37.22 & 37.15 & 37.11 & 37.08 \\
\hline 0.0 & 37.23 & 37.15 & 37.11 & 37.08 \\
\hline-0.005 & 37.22 & 37.15 & 37.11 & 37.08 \\
\hline-0.01 & 37.21 & 37.15 & 37.11 & 37.09 \\
\hline-0.015 & 37.19 & 37.15 & 37.12 & 37.11 \\
\hline-0.02 & 37.18 & 37.15 & 37.13 & 37.12 \\
\hline-0.025 & 37.17 & 37.15 & 37.13 & 37.13 \\
\hline-0.03 & 37.16 & 37.15 & 37.14 & 37.13 \\
\hline-0.035 & 37.16 & 37.15 & 37.14 & 37.14 \\
\hline
\end{tabular}

\section{Procedure for identification of blood perfusion parameters}

GAs are ideally suited to the inverse problem of identifying the parameters used in the temperature-dependence expansions for blood perfusion (Ren et al., 1995; Majchrzak and Paruch, 2004). The method is of an evolutionary type, based on the process of natural selection, requiring no initial guess about the values of the parameters. It is necessary to know only the range of values that these might take in order to choose the number of bits to allocate in each chromosome. No calculations of derivatives, sensitivities or directions in which to search are required.

In this work, a simple GA detailed in Castro and Partridge (2006) has been implemented. The geometry of each tumour is initially considered to be rectangular, of size 0.01 by $0.02 \mathrm{~m}$, with its centre at position $(0.01,0.0)$ as shown in Figure 1 . The size of the initial population of chromosomes is defined considering the range of values which the parameters used to calculate $c_{1}$ in Tables 1 and 2 might take. The highest first-order expansion, curve $\mathrm{F}$, has $c_{1}=800 \times(50+3.0 T)$. If this is written as $c_{1}=800 \times\left(p_{1}+p_{2} T\right)$, then $p_{1}$ will take positive integer values up to 50 and the largest value of $p_{2}$ is 3 , with an interval of 0.1 considered between successive values. Regarding the second-order expansions, $c_{1}=800 \times\left(p_{1}+p_{2} T+p_{3} T^{2}\right), \quad p_{1}$ takes values up to $7, p_{2}$ takes values up to 1.9 , and an interval of 0.1 is considered between successive values. The highest value for parameter $p_{3}$ is 0.07 , with an interval of 0.01 between successive values.

Thus, for the first-order approximation, and considering the values of the coefficients given in Table 1, the chromosome has two alleles; for $p_{1}$ and $p_{2}$ the first allele has 6 bits, $2^{6}-1=63>50$, while the second has 5 bits, in such a way that the 
largest number which can be represented is 3.1 for intervals of 0.1 . For the secondorder approximation, and considering the values of the coefficients given in Table 2, each chromosome has 3 alleles for the values $p_{1}, p_{2}$ and $p_{3}$, the first and third alleles having 3 bits and the second 5 bits. Thus, in both cases, the chromosome is of size 11 . The parameter $p_{1}$ is calculated directly by converting the binary value of the allele to decimal; for $p_{2}$, the decimal value of the allele is divided by 10 , and for $p_{3}$ the decimal value is divided by 100 . The population size was fixed at 20 , which is within the guidelines established by Kahn (2002), who suggested that this value should be between $\ell$ and $2 \ell$ where $\ell$ is the size of the chromosome.

After establishing an initial population, the values of $p_{1}, p_{2}$ and $p_{3}$ for each chromosome are obtained as considered above, and results for the temperature distribution at the skin surface (nodes along the part $\mathrm{AD}$ of the $\Gamma_{2}$ boundary in Figure 1) are calculated using the DRBEM. The objective of the algorithm is to minimise the value of the sum of the squares of the differences between the temperatures calculated at the surface nodes and the predetermined values corresponding to given values of $p_{1}, p_{2}$ and $p_{3}$. The chromosomes are ordered according to the value of the sum of the squares, with the smallest value first, the position in this new order being considered the fitness. Individuals are selected for crossover using the roulette wheel method described by Goldberg (1989), the fitter individuals having the greater probability of selection. The probability of crossover is $80 \%$. Two point crossover and mutation with a probability of $1 \%$ is carried out, following the results in Goldberg et al. (1997). A process of elitism is employed by which the best individual from one generation passes automatically to the next, in order to ensure that the best solution is not lost. A maximum of 100 generations is permitted; if the process has not converged after this, iterations are halted. The stopping criterion considered is that $80 \%$ of the individuals in the fitness table must have converged to the same value. The evolution of the results obtained using the GA is illustrated in the next section for one of the examples considered.

\section{Some results for the identification of perfusion parameters}

Considering the geometry shown in Figure 1, in which a $0.08 \mathrm{~m}$ section of tissue of thickness $0.03 \mathrm{~m}$ is considered with the tumour in the position shown, the temperature values for part $\mathrm{AD}$ of the $\Gamma_{2}$ boundary for the inverse analysis are fixed using the values given in Tables 1 and 2 . These values are compared with those calculated for each tentative set of perfusion parameters indicated by the chromosomes in the GA, and the sum of the squares of the differences minimised. Similar to the direct simulations, the boundary $\Gamma_{2}$ is discretised with 56 linear boundary elements and the boundary $\Gamma_{1}$ with 16 .

\subsection{Results considering a first-order approximation}

It is considered that $c_{1}$ in equation (11) is given by $c_{1}=800 \times\left(p_{1}+p_{2} T\right)$ and the parameters $p_{1}$ and $p_{2}$ are identified. The temperature values used as input on the skin surface are taken from Table 1 .

Case 1. In this case, the data for the measured temperatures on the skin surface are taken from curve A. The results produced by the GA were $p_{1}=1$ and $p_{2}=0.6$, and 
converged in 31 generations. The data used to generate the values at the skin surface are $p_{1}=1$ and $p_{2}=0.5$.

No convergence was obtained for curves B to D as these curves are flat or nearly flat, and the resulting surface temperatures constant or nearly constant.

Case 2. In this case, the data for the measured temperatures on the skin surface are taken from curve E. Although the results are physically unrealistic since the tumour perfusion is too high, it is possible to identify the perfusion parameters due to the surface temperature variations. The results produced by the GA were $p_{1}=31$ and $p_{2}=2.9$, and converged in 21 generations. The data used to generate the values at the skin surface are $p_{1}=30$ and $p_{2}=3$. The evolution of the results obtained using the GA is considered at the end of the section.

Case 3. In this case, the data for the measured temperatures on the skin surface are taken from curve $\mathrm{F}$. The results produced by the GA were $p_{1}=56$ and $p_{2}=2.9$, and converged in 21 generations. The data used to generate the values at the skin surface are $p_{1}=50$ and $p_{2}=3$.

\subsection{Results using a second-order approximation}

It is considered that $c_{1}$ in equation (11) is given by $c_{1}=800 \times\left(p_{1}+p_{2} T+p_{3} T^{2}\right)$ and the parameters $p_{1}, p_{2}$ and $p_{3}$ are identified. The temperature values used as input on the skin surface are taken from Table 2 .

Case 4. In this case, the data for the measured temperatures on the skin surface are taken from curve $\mathrm{G}$. The results produced by the GA were $p_{1}=0, p_{2}=0.6$ and $p_{3}=0.01$. Results converged in 25 generations. The data used to generate the input on the skin surface are $p_{1}=3, p_{2}=0.5$ and $p_{3}=0.01$.

No convergence was obtained for curve $\mathrm{H}$ as this curve is nearly flat, and the resulting surface temperatures nearly constant.

Case 5. In this case, the data for the measured temperatures on the skin surface are taken from curve I. The results produced by the GA were $p_{1}=2, p_{2}=1.2$ and $p_{3}=0.05$. Results converged in 21 generations. The data used to generate the values on the skin surface are $p_{1}=2, p_{2}=1.5$ and $p_{3}=0.04$.

Case 6. In this case, the data for the measured temperatures on the skin surface are taken from curve J. The results produced by the GA were $p_{1}=2, p_{2}=2.1$ and $p_{3}=0.07$. Results converged in 32 generations. The data used to generate the values on the skin surface are $p_{1}=7, p_{2}=1.9$ and $p_{3}=0.07$.

\subsection{Evolution of $G A$ results for Case 2}

As the initial population in the GA is generated randomly, the predicted values of $p_{1}$ and $p_{2}$ display a large scatter, as shown in Figure 2a. There is already considerable improvement after 5 generations (Figure 2b), and much improvement after 10 generations (Figure 2c), with $70 \%$ of chromosomes predicting the solution $p_{1}=30$, 
$p_{2}=2.9$. The process is considered to have converged after 21 generations (Figure $2 \mathrm{~d}$ ), with $85 \%$ of the solutions producing the same values $p_{1}=31, p_{2}=2.9$, compared to the correct solution $p_{1}=30, p_{2}=3$. In this case, however, we extended the simulations to obtain convergence of $100 \%$ of the chromosomes; the converged values, $p_{1}=31, p_{2}=2.9$, were obtained after 36 generations (Figure 2e).

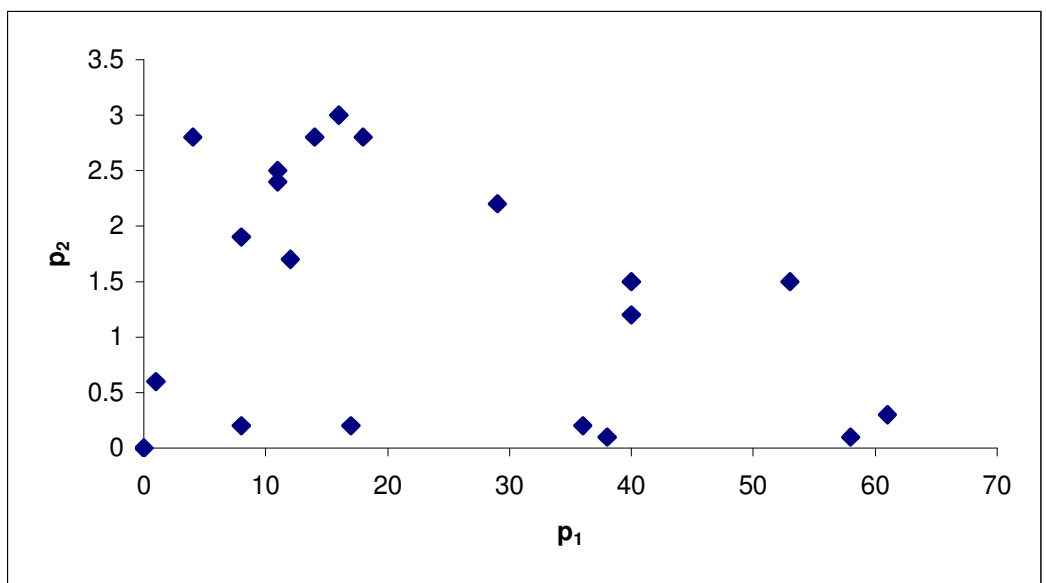

a. Initial Population

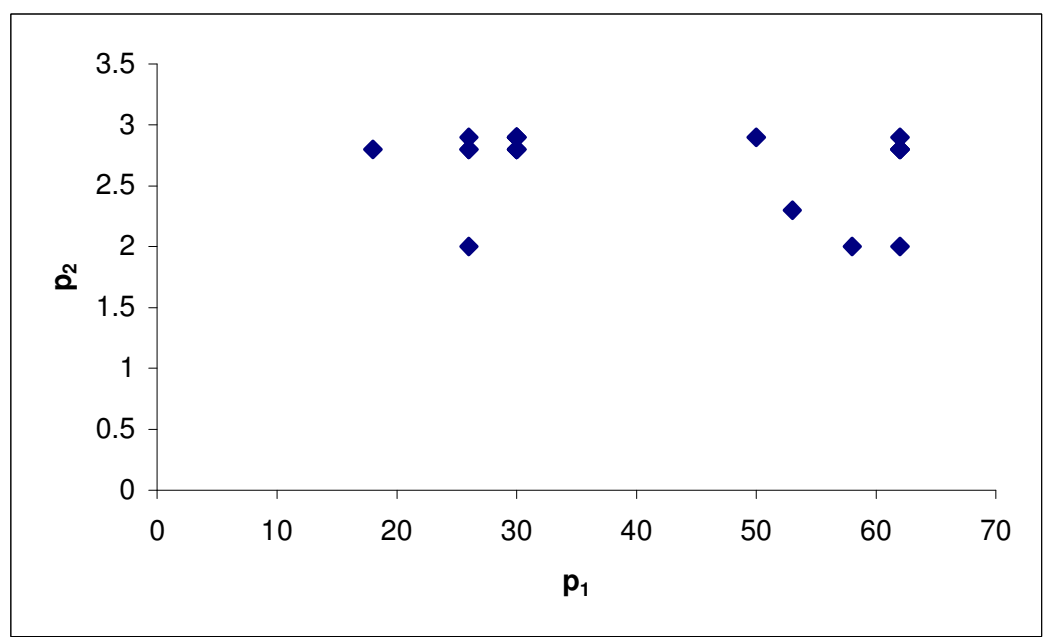

b. Generation 5 


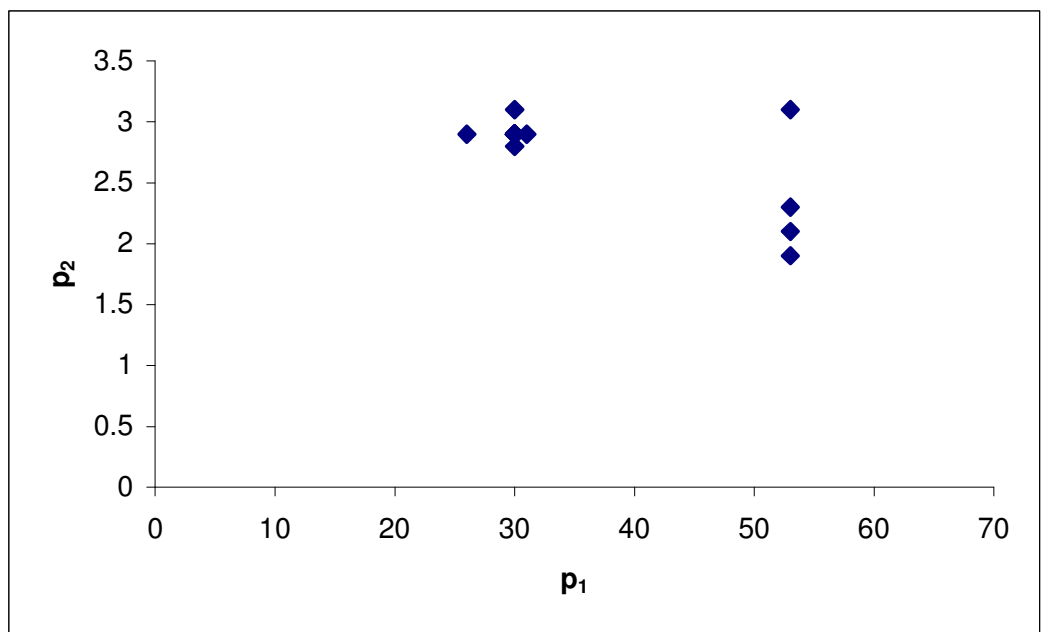

c. Generation 10

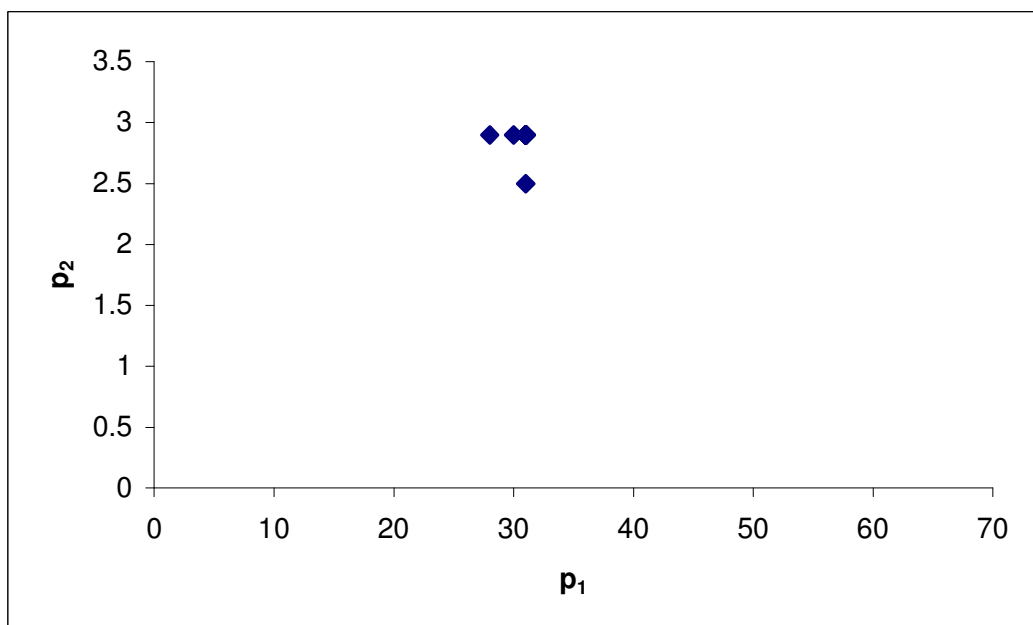

d. Generation 21

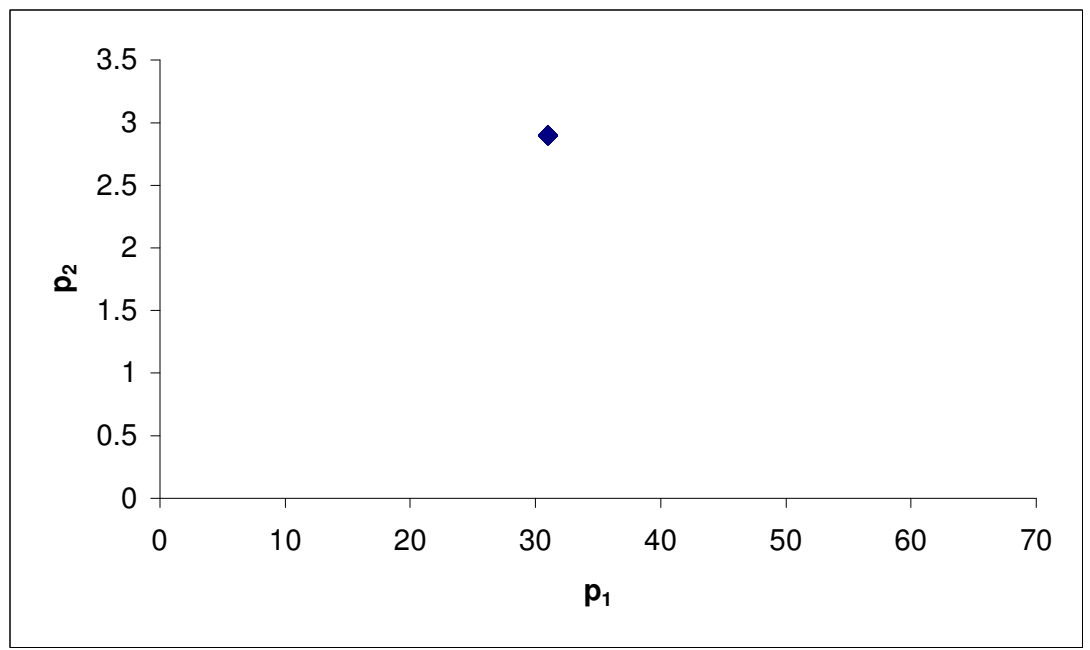

e. Generation 36

Figure 2. Evolution of GA results for curve $\mathrm{E}$ in Table 1 


\subsection{Results for a circular tumour}

A circular tumour of radius 0.01 was next analysed. The centre of the tumour was located at position $(0.015,0.0)$, and its discretisation used 16 nodes. The total discretisation thus has the same number of nodes and elements as for the rectangular tumours.

Initially, a direct simulation was carried out to obtain the temperature distribution at the skin surface using first-order tumour perfusion. Results for three different cases are shown in Table 3 . The curve labels refer to the variations defined in Table 1.

Table 3: Temperature variations $\left({ }^{\circ} \mathrm{C}\right)$ at the skin surface for a circular tumour considering first-order perfusion

\begin{tabular}{|l|l|l|l|}
\hline$y$ at skin surface & Curve A & Curve E & Curve F \\
\hline 0.035 & 37.17 & 37.14 & 37.14 \\
\hline 0.03 & 37.18 & 37.14 & 37.13 \\
\hline 0.025 & 37.19 & 37.13 & 37.13 \\
\hline 0.02 & 37.21 & 37.13 & 37.12 \\
\hline 0.015 & 37.24 & 37.12 & 37.11 \\
\hline 0.01 & 37.27 & 37.11 & 37.10 \\
\hline 0.005 & 37.31 & 37.10 & 37.09 \\
\hline 0.0 & 37.32 & 37.09 & 37.08 \\
\hline-0.005 & 37.31 & 37.10 & 37.09 \\
\hline-0.01 & 37.27 & 37.11 & 37.10 \\
\hline-0.015 & 37.24 & 37.12 & 37.11 \\
\hline-0.02 & 37.21 & 37.13 & 37.12 \\
\hline-0.025 & 37.19 & 37.13 & 37.13 \\
\hline-0.03 & 37.18 & 37.14 & 37.13 \\
\hline-0.035 & 37.17 & 37.14 & 37.14 \\
\hline
\end{tabular}

It can be seen, by comparison with Table 1, that the results differ little from those for a rectangular tumour.

For the inverse analysis, data for the measured temperatures on the skin surface are initially taken from curve $A$ in Table 3 . The results produced by the GA were $p_{1}=0$ and $p_{2}=0.6$, and converged in 31 generations. The data used to generate the values at the skin surface are $p_{1}=1$ and $p_{2}=0.5$.

Next, skin temperature data are taken from curve E. The results produced by the GA were $p_{1}=25$ and $p_{2}=3.1$, and converged in 43 generations. The data used to generate the values at the skin surface are $p_{1}=30$ and $p_{2}=3$. Finally, skin temperature data are taken from curve $F$. The results produced by the GA were $p_{1}=47$ and $p_{2}=3.0$, and also converged in 43 generations. The data used to generate the values at the skin surface are $p_{1}=50$ and $p_{2}=3$.

\section{Conclusions}

In this paper, the DRBEM was coupled to a GA in an inverse procedure for identifying parameters of a temperature-dependent approximation for the tumour perfusion, considering temperature data on the skin surface. The procedure has the advantage of not requiring the calculation of derivatives or sensitivities, or an initial 
estimate of these values. The DRBEM formulation requires no internal discretisation, and in this case no internal nodes either, apart from those defining the interface tissue/tumour. The technique can be directly extended to more realistic threedimensional inverse analysis estimations, at an increased computational cost.

It is seen that the skin temperature variation changes as the blood perfusion increases, and in certain cases flat or nearly flat curves are produced. The proposed algorithm has difficulty to identify the perfusion parameters in these cases, although a more advanced GA may provide improved results.

Hyperthermia cancer treatment normally involves heating tissue to $42-43^{\circ} \mathrm{C}$. This is achieved through the use of electromagnetic waves, and should be designed to avoid 'hot spots' in healthy tissue and 'cold spots' in the tumour region. When heated up to this range of temperatures, the blood flow in normal tissues, e.g. skin and muscle, increases significantly, while blood flow in the tumour zone decreases (Erdmann et al., 1998). Therefore, the consideration of temperature-dependent blood perfusion in this case is not only essential for the correct modelling of the problem, but should provide larger skin temperature variations, making the identification problem easier.

\section{Acknowledgement}

The first author gratefully acknowledges the support of the Brazilian agency CAPES in funding this work.

\section{References}

Bridges, T.R. and Wrobel, L.C. (1996), "A DRM formulation for elasticity problems with body forces using augmented thin plate splines", Communications in Numerical Methods in Engineering, Vol. 12, pp. 209-220.

Castro, L.C. and Partridge, P.W. (2006), "Minimum weight design of framed structures using a genetic algorithm considering dynamic analysis", Latin American Journal of Solids and Structures, Vol. 3, pp. 107-123.

Deng, Z-S. and Liu, J. (2000), "Parametric studies on the phase shift method to measure the blood perfusion of biological bodies", Medical Engineering \& Physics, Vol. 22, pp. 693-702.

Deng, Z-S. and Liu, J. (2004a), "Mathematical modelling of temperature mapping over skin surface and its implementation in thermal disease diagnostics", Computers in Biology and Medicine, Vol. 34, pp. 495-521.

Deng, Z-S. and Liu, J. (2004b) "Modelling of multidimensional freezing problem during cryosurgery by the dual reciprocity boundary element method", Engineering Analysis with Boundary Elements, Vol. 28, pp. 97-108.

Erdmann, B., Lang, J. and Seebass, M. (1998), "Optimization of temperature distributions for regional hyperthermia based on a nonlinear heat transfer model", Annals of the New York Academy of Sciences, Vol. 858, pp. 36-46. 
Golberg, M.A. and Chen, C.S. (1994), "The theory of radial basis functions applied to the BEM for inhomogeneous partial differential equations", Boundary Element Communications, Vol. 5, pp. 57-61.

Goldberg, D.E. (1989), Genetic Algorithms in Search, Optimization and Machine Learning, Addison-Wesley, Boston.

Goldberg, D.E., Zakrewsky, K., Sutton, B., Gadient, R., Chong, C., Galego, P., Miller, B. and Cantu-Paz, E. (1997), "Genetic algorithms: A bibliography", Technical Report 97011, University of Illinois at Urbana-Champaign.

Kahn, N. (2002), "Population sizing in genetic and evolutionary algorithms", Internal Report, University of Illinois at Urbana Champaign.

Lang, J., Erdmann, B. and Seebass, M. (1999), "Impact of nonlinear heat transfer on temperature control in regional hyperthermia", IEEE Transactions on Biomedical Engineering, Vol. 46, pp. 1129-1138.

Liu, J. and Xu, L.S. (2000), "Boundary information based diagnostics on the thermal states of biological bodies", International Journal of Heat and Mass Transfer, Vol. 43, pp. 2827-2839.

Lu, W-Q., Liu, J., and Zeng, Y. (1998), "Simulation of the thermal wave propagation in biological tissues by the dual reciprocity boundary element method", Engineering Analysis with Boundary Elements, Vol. 22, pp. 167-174.

Majchrzak, E. and Paruch, M. (2004), "Identification of the tumour region on the basis of skin surface temperature", Proceedings ECCOMAS 2004, Eds P. Neittaanmaki et al., pp. 1-14.

Partridge, P.W. (2000), "Towards criteria for selecting approximation functions in the dual reciprocity method", Engineering Analysis with Boundary Elements, Vol. 24, pp. 519-529.

Partridge, P.W., Brebbia, C.A. and Wrobel, L.C. (1992), The Dual Reciprocity Boundary Element Method, Elsevier, London.

Partridge, P.W. and Wrobel, L.C. (2007), "An inverse geometry problem for the localisation of skin tumours by thermal analysis", Engineering Analysis with Boundary Elements, Vol. 31, pp. 803-811.

Rai, K.N. and Rai, S.K. (1999), "Effect of metabolic heat generation and blood perfusion on the heat transfer in tissues with a blood vessel", Heat and Mass Transfer, Vol. 35, pp. 75-79.

Ren, Z.P., Liu, J. and Wang, C.C. (1995), "Boundary element method for solving normal or inverse bio-heat transfer problems of biological bodies with complex shape", Journal of Thermal Sciences, Vol. 4, pp. 117-124. 
Tompkins, D.T., Vanderby, R., Klein, S.A., Beckman, W.A., Steeves, R.A., Frye, D.M. and Paliwal, B.R. (1994), "Temperature-dependent versus constant-rate blood perfusion modelling in ferromagnetic thermoseed hyperthermia: results with a model of the human prostate", International Journal of Hyperthermia, Vol. 10, pp. 517-536. 